\title{
Diversity matters
}

\author{
For genomics - and science in general — to fulfil its potential of supporting efforts to understand \\ disease and improve human health, diversity and inclusion must be recognized as essential \\ catalysts of research success.
}

\section{Cf Diversity is a catalyst for innovation and helps us grow פק}

Awareness is growing of the importance of including diverse, under-represented and underserved individuals across the research cycle, from increasing participant diversity in large population studies to recruiting a more diverse workforce ${ }^{1}$. A commitment to improving all types of diversity - from gender and ethnicity to socioeconomic background and neurodiversity - across genomics research is needed, not least because it is the right thing to do but also because diversity and inclusion are necessary catalysts of innovation that drive the success of scientific research enterprises.

As highlighted by Gurdasani et al. ${ }^{2}$ in this issue, genome-wide association studies (GWAS) listed in the NHGRI-EBI GWAS Catalog are currently heavily biased towards individuals of European ancestries. This bias comes at a cost. Which and how genetic variants affect disease can vary across populations, and results obtained in one population may thus not be transferable to another. Indeed, a study published in Nature $^{3}$ recently confirmed that genetic variant effect sizes and polygenic risk prediction scores derived from one population cannot be readily applied to populations of different ancestry, as summarized in a recent Research Highlight ${ }^{4}$. Increasing the inclusion of research participants from populations that have been traditionally under-represented in genome sequencing or genetic association studies has led to the discovery of new causal variants and disease associations, helping to identify potential differences between populations. Not only will such an approach further our ability to understand the genetic contributions to health and disease, it will also ensure a more equitable distribution of the benefits that genomics can bring.

Improving recruitment and participation in genomic studies (or scientific research in general) among underrepresented groups can be difficult, for example, as a result of historical mistrust of research endeavours. As emphasized in a Comment by Tsosie et al. ${ }^{5}$, researchers need to carefully consider how they engage with the minority communities they aim to help. For example, recruiting Indigenous individuals residing in urban centres without tribal consultation may be less timeconsuming and resource-intensive than engaging tribes; however, given the small size of many Indigenous populations, individual consent models can pose risks to the community as a whole.

One way to ensure that genomic research better serves the needs of diverse and under-represented communities is through diversification of the research workforce itself, whereby “'the researched' must now become 'the researchers' who set the agenda" ${ }^{\text {" }}$. Bringing together different kinds of people to look at problems or data from different perspectives is likely to widen the focus and the types of research question being pursued. To increase diversity in the workforce, more equitable pathways into research will be needed, and barriers to a fully diverse workforce, such as unconscious bias and stereotypical thinking, will need to be overcome.

If the above fails to incentivize prioritizing diversity in future strategies, readers may be encouraged by evidence connecting diversity and scientific reach and business impact. Companies that engage effectively with diversity and inclusion have been shown to gain a competitive advantage, with improvements seen across recruitment, global reputation, and customer and employee satisfaction, among others ${ }^{7}$.

Despite convincing evidence of its benefits, increasing diversity in genomics and effectively using it to make an impact is not straightforward. The time and commitment needed for change should not be underestimated but will be imperative to build a solid foundation for genomic research and medicine. Diversity is a catalyst for innovation and helps us grow. Valuing diversity and striving for inclusion will allow genetic discovery and precision medicine to benefit everyone.

1. Hindorff, L. A. et al. Nat. Rev. Genet. 19, 175-185 (2018).

2. Gurdasani, D. et al. Nat. Rev. Genet. https://doi.org/10.1038/ s41576-019-0144-0 (2019)

3. Wojcik, G. L. et al. Nature 570, 514-518 (2019).

4. Clyde, D. Nat. Rev. Genet. https://doi.org/10.1038/ s41576-019-0160-0 (2019).

5. Tsosie, K. S., Yracheta, J. M. \& Dickenson, D. Nat. Rev. Genet. https://doi.org/10.1038/s41576-019-0161-z (2019).

6. Powell, K. Nature 558, 19-22 (2018).

7. Hunt, V. et al. McKinsey \& Company https://www. mckinsey.com/ business-functions/organization/our-insights/delivering-throughdiversity (2018). 